\title{
Synthesis, structures and anti-HBV activities of derivatives of the glutarimide antibiotic cycloheximide
}

\author{
Hui-fang Guo, Yu-huan Li, Hong Yi, Tian Zhang, Shu-qin Wang, Pei-zhen Tao and Zhuo-rong Li
}

The Journal of Antibiotics (2009) 62, 639-642; doi:10.1038/ja.2009.87; published online 28 August 2009

Keywords: anhydrocycloheximide; antiviral activity; crystal structure; cycloheximide

Cycloheximide (CHX) (Figure 1), produced by Streptomyces griseus, is a heterocyclic glutarimide antibiotic. It inhibits protein synthesis in most eukaryotic cells and is a well-known tool commonly used in biomedical research. ${ }^{1}$ CHX has been reported to significantly inhibit both simian virus $40 \mathrm{~T}$ antigen and cellular DNA synthesis in CV-1 cells. ${ }^{2}$ Research efforts also showed that CHX inhibited RNA production of influenza $\mathrm{A},{ }^{3}$ reovirus ${ }^{4}$ and arbovirus, ${ }^{5}$ and also inhibited hepatitis A virus replication. ${ }^{6}$ Recent reports have shown that $\mathrm{CHX}$ contributes to the control of human immunodeficiency virus (HIV) by modifying viral protein ratios. ${ }^{7,8}$ This observation indicates that CHX may represent an anti-HIV candidate. In this study, the inhibitory activity of CHX against hepatitis B virus (HBV) in 2.2.15 cells was investigated. The results showed that the anti-HBV activity of $\mathrm{CHX}\left(\mathrm{IC}_{50}=45.5 \mu \mathrm{g} \mathrm{ml}^{-1}\right.$ ) was weaker than that of 3TC (Lamivudine) (Table 1). Dehydration of CHX resulted in the formation of two compounds (1a and $\mathbf{1 b}$ ), which were separated by high pressure liquid chromatography. Compounds $\mathbf{1 a}$ and $\mathbf{1 b}$ significantly inhibited DNA replication of $\mathrm{HBV}$ and the $\mathrm{IC}_{50}$ values were 1.67 and $2.14 \mu \mathrm{g} \mathrm{ml}^{-1}$, respectively, with high selectivity indexes (93.0 and 31.1 , respectively). The structure of $\mathbf{1 a}$ was elucidated by X-ray diffraction, and that of $\mathbf{1} \mathbf{b}$ was confirmed as the $3^{\prime \prime} S$ epimer of $\mathbf{1 a}$ by CD spectroscopy.

Compounds $\mathbf{1 a}$ and $\mathbf{1 b}$ were produced as a mixture from $\mathrm{CHX}$ by dehydrating in dichloromethane with $\mathrm{BF}_{3} \mathrm{OEt}_{2}$ as the catalyst. Schaeffer and Jain ${ }^{9}$ have predicted that the methyl groups in CHX's dehydration product (often known as anhydrocycloheximide) exist as a mixture of both cis and trans forms. However, no previous study has confirmed whether the geometric structure of the $\mathrm{C}=\mathrm{C}$ bond in anhydrocycloheximide is $Z$ or $E$. The stereo structure (Figure 2) of 1a was determined by X-ray diffraction, in which C-3" (C10 in Figure $2 \mathrm{~b}$ ) was found to be $R$, and the double bond between $\mathrm{C}-2^{\prime}$ and C- $1^{\prime \prime}$ (C6 and C4 in Figure 2b) was the E configuration. Crystallographic data for the structure of 1a have been deposited in the Cambridge Crystallographic Data Center with deposition no. CCDC719670.
The C- 3 " of CHX was the $S$ configuration, whereas the configuration of $\mathrm{C}-3^{\prime \prime}$ of $\mathbf{1 a}$ was $R$. Consequently, we deduced that $\mathbf{1 b}$ must be the epimer of la with C- 3 " in the $S$ configuration based on ${ }^{1} \mathrm{H}$ NMR, ${ }^{13} \mathrm{C}$ NMR, MS, IR and NOE spectra. In the NOE experiments, irradiation of $1^{\prime}-\mathrm{H}$ of $\mathbf{1 b}$ enhanced the intensity of $6^{\prime \prime}-\mathrm{H}_{\beta}$, and the irradiation of $1^{\prime}-\mathrm{H}$ of $1 \mathrm{a}$ also enhanced the intensity of $6^{\prime \prime}-\mathrm{H}_{\beta}$. Moreover, irradiation of $2^{\prime}-$ $\mathrm{H}$ of compound $\mathbf{1 a}$ or $\mathbf{1 b}$ enhanced the intensity of $1^{\prime}-\mathrm{H}$; however, no enhancement of intensity of $6^{\prime \prime}-\mathrm{H}$ of $\mathbf{1 a}$ or $\mathbf{1 b}$ was observed. The NOE data for $\mathbf{1 b}$ and $\mathbf{1 a}$ indicated that the configuration of the double bond of $\mathbf{1 b}$ was the same as that of $\mathbf{1 a}$, which was the $E$ configuration.

To confirm that $\mathbf{1 b}$ is the $3^{\prime \prime} S$ configuration, CD spectra of compounds $\mathbf{1 a}$ and $\mathbf{1 b}$ were acquired (Figure 3 ). The CD spectra of $\alpha, \beta$-unsaturated ketones usually show three CD bands above $180 \mathrm{~nm}$. The two $\mathrm{C}=\mathrm{C} \pi \rightarrow \pi^{\star}$ bands $(200-260 \mathrm{~nm})$ of $\mathbf{1 a}$ and $\mathbf{1 b}$ were similar, but the C-2" keto $n \rightarrow \pi^{\star}$ bands $(260-400 \mathrm{~nm})$ of $\mathbf{1 a}$ and $\mathbf{1 b}$ were opposite. Clearly, 1a showed a negative Cotton effect, whereas $\mathbf{1 b}$ showed a positive Cotton effect. Both maximum absorption wavelengths of $\mathbf{1 a}$ and $\mathbf{1 b}$ were near $320 \mathrm{~nm}$ (1a: $327.5 \mathrm{~nm}$, $\left.\Delta \varepsilon=-0.88 \mathrm{~cm}^{2} \mathrm{mmol}^{-1} ; \quad \mathbf{l b}: 323 \mathrm{~nm}, \Delta \varepsilon=0.44 \mathrm{~cm}^{2} \mathrm{mmol}^{-1}\right)$. The difference in the $\mathrm{CD}$ behavior of the $\mathrm{C}-2^{\prime \prime}$ keto originated from the different chirality of the C- $3^{\prime \prime}$. In conclusion, the data showed that $\mathbf{1 b}$ is 4 - $\left[2^{\prime}-\left(3^{\prime \prime}(S), 5^{\prime \prime}(S)-3^{\prime \prime}, 5^{\prime \prime}\right.\right.$-dimethyl-2" -oxocyclohexylidene)ethyl] piperidine-2,6-dione. During the dehydration process of CHX, ketoenol tautomerization involving the $\mathrm{C}-2^{\prime \prime}$ keto and the $\mathrm{C}-3^{\prime \prime}$ proton leads to epimerization at the $\mathrm{C}-3^{\prime \prime}$. On the basis of quantitative analysis with HPLC, the production of 1 a was $\sim 1.8$ times greater than that of $\mathbf{1 b}$. Consequently, $\mathbf{1 a}$ seems to be more stable than does $\mathbf{1 b}$. The total energies of compounds $\mathbf{1 a}$ and $\mathbf{1 b}$ were compared on the basis of calculations using SYBYL (version 7.35, Tripos, St Louis, MO, USA). The results support our hypothesis as the calcd total energy for 1a was $-6.053 \mathrm{kcal} \mathrm{mol}^{-1}$ and that of $\mathbf{1 b}$ was $-5.747 \mathrm{kcal} \mathrm{mol}^{-1}$.

Alkylation of the nitrogen atom of the glutarimide ring of $\mathbf{1 a}$ afforded compounds 2 and 3 . Reduction of the keto group of 1a produced compound 4 (Scheme 1). Compounds 2, 3 and 4 did not inhibit the growth of HBV. 
<smiles>C[C@H]1C[C@H](C)C(=O)[C@@H]([C@@H](O)C=C2CC(=O)NC(=O)C2)C1</smiles>

Figure 1 Structure of cycloheximide (CHX).

Table 1 Anti-HBV activities of $\mathrm{CHX}$ analogs

\begin{tabular}{lccccccc}
\hline Compounds & CHX & $\mathbf{1 a}$ & $\mathbf{1 b}$ & $\mathbf{2}$ & $\mathbf{3}$ & $\mathbf{4}$ & 3TC $^{a}$ \\
\hline $\mathrm{IC}_{50}\left(\left.\mu \mathrm{g} \mathrm{m}\right|^{-1}\right)$ & 45.5 & 1.67 & 2.14 & $>200$ & $>200$ & $>200$ & 0.89 \\
$\mathrm{SI}^{\mathrm{b}}$ & 4.4 & 93.0 & 31.1 & $/$ & $/$ & $/$ & 1315 \\
\hline
\end{tabular}

Abbreviations: $\mathrm{CHX}$, cycloheximide; HBV, hepatitis B virus; SI, selective index. a3TC: Lamivudine, an antiviral agent used as positive control.

${ }^{\mathrm{b}} \mathrm{S} \mathrm{I}=\mathrm{TC}_{50} / \mathrm{IC}_{50}$

a<smiles>C[C@@H]1CC(=CCC2CC(=O)NC(=O)C2)C(=O)[C@@H](C)C1</smiles>

The molecular structure of $1 \mathrm{a} \quad$ X-ray crystal structure of $1 \mathrm{a}$

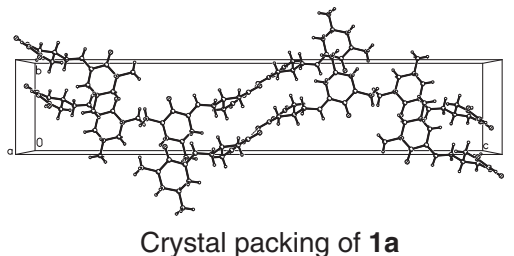

Figure 2 X-ray structures of 1 a. (a) The molecular structure of $1 \mathrm{a}$. (b) X-ray crystal structure of $1 \mathrm{a}$. (c) Crystal packing of $1 \mathrm{a}$.

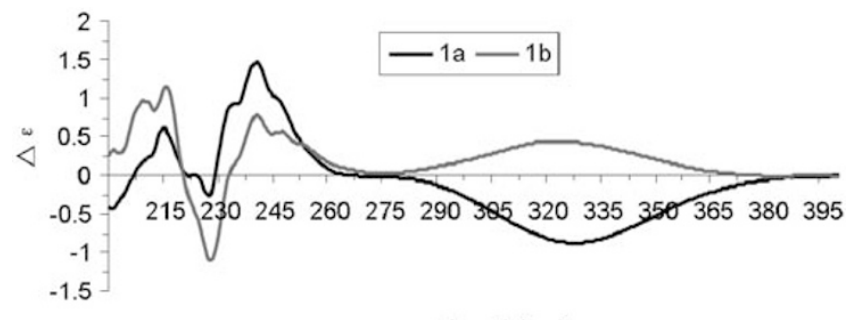

wavelength $(\mathrm{nm})$

Figure $3 \mathrm{CD}$ spectra of $1 \mathbf{a}$ and $\mathbf{1 b}$ (in chloroform, $c=3.8 \times 10^{-3} \mathrm{M}$, at $25^{\circ} \mathrm{C}$ ).

In summary, CHX is a weak inhibitor of HBV in 2.2 .15 cells. Under acid-catalyzed dehydration conditions in dichloromethane, the stereo configuration of the C- $3^{\prime \prime}$ methyl group in the cyclohexanone ring of CHX was epimerized and the two epimers $\mathbf{1 a}$ and $\mathbf{1 b}$ were formed. Both $\mathbf{1 a}$ and $\mathbf{1 b}$ showed much stronger anti-HBV activities (more than 20-fold higher) and higher selectivity indexes than did CHX. The absolute configuration of compound 1a was determined by X-ray

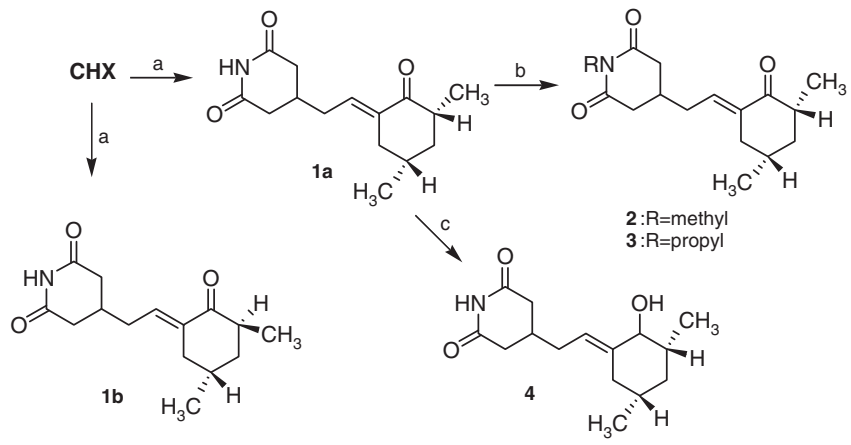

Scheme 1 Reagents and conditions: (a) $\mathrm{BF}_{3} \mathrm{OEt}_{2}, \mathrm{CH}_{2} \mathrm{Cl}_{2}$; (b) $\mathrm{CH}_{3} \mathrm{I}$ or $\mathrm{CH}_{3} \mathrm{CH}_{2} \mathrm{CH}_{2} \mathrm{I}, \mathrm{K}_{2} \mathrm{CO}_{3}, \mathrm{CH}_{3} \mathrm{CN}, 65^{\circ} \mathrm{C}$; (c) $\mathrm{Zn}\left(\mathrm{BH}_{4}\right)_{2}-\mathrm{DME}$ solution, DME. DME, 1,2-dimethoxyethane.

diffraction, and the structure of $\mathbf{1 b}$ was determined on the basis of ${ }^{1} \mathrm{H}$ NMR, ${ }^{13} \mathrm{C}$ NMR, NOE and CD spectra. Alkylation of the nitrogen atom of the glutarimide ring of $\mathbf{1 a}$ and reduction of the keto group of 1a lead to the loss of anti-HBV activity. These results showed that the double bond between $\mathrm{C}-2^{\prime}$ and $\mathrm{C}-1^{\prime \prime}$, the $\mathrm{NH}$ proton and the $2^{\prime \prime}$ carbonyl group in the structure might be essential for anti-HBV activity.

\section{EXPERIMENTAL SECTION}

\section{General methods}

Melting points were determined using an $\mathrm{X}_{6}$ microscope melting point apparatus and were uncorrected. ${ }^{1} \mathrm{H}$ NMR, ${ }^{13} \mathrm{C}$ NMR spectra and NOE data were recorded on a Varian Mercury-400 spectrometer (Varian, Beijing, China). HRMS spectra were recorded on an AccuTOF CS mass spectrometer (AccuTOF, Jeol Ltd, Beijing, China). HPLC was performed using a Shimadzu LC-10Avp with a SPD-10Avp UV detector (Shimadzu, Beijing, China) and a Class VP 6.x Workstation (Shimadzu). The column used was a Diamonsil C18 $5-\mu \mathrm{m} 250 \times 4.6 \mathrm{~mm}$ column (Dikma Technologies, Beijing, China). Preparative liquid chromatography was performed using a Shimadzu LC-6AD $v p$ liquid chromatograph instrument (Shimadzu) with a SPD-10Avp UV detector and Class VP 6.x workstation. The column used was a Shim-pack PREP-ODS $20 \mathrm{~mm} \mathrm{ID} \times 25 \mathrm{~cm}$ column (Shimadzu) with a 100 - $\AA$ pore diameter and $15-\mu \mathrm{m}$ particle diameter. Optical rotations were measured on a Perkin Elmer Model 341 LC parameter (Perkin Elmer, Shanghai, China). X-ray data were collected on a MAC DIP-2030K diffractometer. Circular dichroism spectra were recorded on a Jasco J-815 CD spectropolarimeter (Jasco, Tokyo, Japan).

\section{4-[2'-( $3^{\prime \prime}(R), 5^{\prime \prime}(S)-3^{\prime \prime}, 5^{\prime \prime}$-dimethyl-2" -oxocyclohexylidene)ethyl]} piperidine-2,6-dione (1a) and $4-\left[2^{\prime}-\left(3^{\prime \prime}(S), 5^{\prime \prime}(S)-3^{\prime \prime}, 5^{\prime \prime}\right.\right.$-dimethyl$2^{\prime \prime}$-oxocyclohexylidene)ethyl]piperidine-2,6-dione (1b)

$\mathrm{BF}_{3} \mathrm{OEt}_{2}(1.76 \mathrm{ml}, 1.4 \mathrm{mmol})$ was added slowly to an ice-salt cold solution of CHX $(2.0 \mathrm{~g}, 7 \mathrm{mmol})$ in $20 \mathrm{ml}$ of dichloromethane. After stirring for $5 \mathrm{~min}$, the salt ice-bath was withdrawn and stirring was continued for $3 \mathrm{~h}$ at room temperature. The ice-bath was used at the end of the stirring period. The solution was quenched with $20 \mathrm{ml}$ of saturated $\mathrm{NaHCO}_{3}$ solution when the temperature of the solution reached $0{ }^{\circ} \mathrm{C}$. The water layer was extracted with dichloromethane $(20 \mathrm{ml} \times 3)$. The combined organic layer was washed with $60 \mathrm{ml}$ of saturated $\mathrm{NaCl}$ and $60 \mathrm{ml}$ of water and then dried over anhydrous magnesium sulfate. The solution was filtered to remove the precipitate and the filtrate was concentrated in vacuo. The residue was purified by chromatography using a silica gel column (100:15 dichloromethane/acetone) to afford a mixture of the compounds $1 \mathbf{a}$ and $1 \mathbf{b}(1.10 \mathrm{~g}$, yield $55 \%)$ as a white powder.

Compounds $\mathbf{1 a}$ and $\mathbf{1 b}$ were separated by preparative liquid chromatography. The chromatography was performed on a Shim-pack PREP-ODS at $25^{\circ} \mathrm{C}$ with a mobile phase of water-methanol 50:50 (v/v). The flow rate was $10 \mathrm{ml} \mathrm{min}^{-1}$, and detection was achieved by monitoring the UV absorbance at $231 \mathrm{~nm}$. The sample was dissolved in dimethyl sulfoxide, and sample-loading volumes were $100 \mu$ l. The separated solution was concentrated until no 
methanol existed and was then dried by vacuum freeze drying. Compounds 1a and $\mathbf{1 b}$ were isolated and both appeared as a white powder.

Physical data for compound 1a: m.p. $130-131^{\circ} \mathrm{C} ;[\alpha]_{\mathrm{D}}^{20}=-30.9^{\circ} \mathrm{C}(c=1.25$, $\left.\mathrm{CHCl}_{3}\right)$; IR: 3189, $2930(\mathrm{NH}), 1712(\mathrm{C}=\mathrm{O}), 1682(\mathrm{C}=\mathrm{O}) \mathrm{cm}^{-1} ;{ }^{1} \mathrm{H}$ NMR $\left(400 \mathrm{MHz}, \mathrm{CDCl}_{3}\right) \delta: 1.05\left(3 \mathrm{H}, \mathrm{d}, J=5.6 \mathrm{~Hz}, 5^{\prime \prime}-\mathrm{Me}\right), 1.13(3 \mathrm{H}, \mathrm{d}, J=6.8 \mathrm{~Hz}$, $\left.3^{\prime \prime}-\mathrm{Me}\right), 1.22-1.32\left(1 \mathrm{H}, \mathrm{m}, 4^{\prime \prime}-\mathrm{H}_{\alpha}\right), 1.84-1.93\left(2 \mathrm{H}, \mathrm{m}, 5^{\prime \prime}-\mathrm{H}, 6^{\prime \prime}-\mathrm{H}_{\alpha}\right)$, $1.96-2.01\left(1 \mathrm{H}, \mathrm{m}, 4^{\prime \prime}-\mathrm{H}_{\beta}\right), 2.16-2.25\left(2 \mathrm{H}, \mathrm{m}, 1^{\prime}-\mathrm{H}\right), 2.27-2.38(4 \mathrm{H}, \mathrm{m}$, $\left.3^{\prime \prime}-\mathrm{H}, 4-\mathrm{H}, 3-\mathrm{H}_{\alpha}, 5-\mathrm{H}_{\alpha}\right), 2.67-2.73\left(3 \mathrm{H}, \mathrm{m}, 6^{\prime \prime}-\mathrm{H}_{\beta}, 3-\mathrm{H}_{\beta}, 5-\mathrm{H}_{\beta}\right), 6.35(1 \mathrm{H}, \mathrm{t}$, $J=8.0 \mathrm{~Hz},=\mathrm{CH}), 7.85(1 \mathrm{H}, \mathrm{br}, \mathrm{NH}) ;{ }^{13} \mathrm{C} \mathrm{NMR}\left(100 \mathrm{MHz}, \mathrm{CDCl}_{3}\right): 15.7\left(3^{\prime \prime}-\right.$ Me), 22.1 (5"-Me), 30.1 (C-4), 30.3 (C-5"), 32.4 (C-1'), 36.2 (C-6"), 37.4 (C-3), 37.4 (C-5), $40.7\left(\mathrm{C}-4^{\prime \prime}\right), 44.1$ (C-3'), $131.6\left(\mathrm{C}-2^{\prime}\right), 139.3\left(\mathrm{C}-1^{\prime \prime}\right), 171.5(\mathrm{C}-2$, C-6), $203.2\left(\mathrm{C}-2^{\prime \prime}\right)$; MS $\left(\mathrm{ESI}^{+}\right) \mathrm{m} / z: 286.4[\mathrm{M}+\mathrm{Na}]^{+}$, HRMS $\left(\mathrm{ESI}^{+}\right) \mathrm{m} / \mathrm{z}$ : $286.14183[\mathrm{M}+\mathrm{Na}]^{+}$, calcd mass for $\mathrm{C}_{15} \mathrm{H}_{21} \mathrm{NO}_{3} \mathrm{Na}$ : 286.14191.

Physical data for compound lb: m.p. $118-120^{\circ} \mathrm{C} ;[\alpha]_{\mathrm{D}}^{20}=+45.7^{\circ} \mathrm{C}(c=1.03$, $\left.\mathrm{CHCl}_{3}\right)$; IR: 3201, $2927(\mathrm{NH}), 1721(\mathrm{C}=\mathrm{O}), 1678(\mathrm{C}=\mathrm{O}) \mathrm{cm}^{-1}$; $1 \mathrm{H} \mathrm{NMR}$ $\left(400 \mathrm{MHz}, \mathrm{CDCl}_{3}\right) \delta: 1.04\left(3 \mathrm{H}, \mathrm{d}, J=6.4 \mathrm{~Hz}, 5^{\prime \prime}-\mathrm{Me}\right), 1.12(3 \mathrm{H}, \mathrm{d}, J=7.2 \mathrm{~Hz}$, $\left.3^{\prime \prime}-\mathrm{Me}\right), 1.64-1.77\left(2 \mathrm{H}, \mathrm{m}, 4^{\prime \prime}-\mathrm{H}\right), 2.01-2.11\left(2 \mathrm{H}, \mathrm{m}, 4-\mathrm{H}, 6^{\prime \prime}-\mathrm{H}_{\alpha}\right), 2.19-2.21$ $\left(2 \mathrm{H}, \mathrm{m}, \mathrm{1}^{\prime}-\mathrm{H}\right), 2.28-2.31\left(3 \mathrm{H}, \mathrm{m}, 5^{\prime \prime}-\mathrm{H}, 3-\mathrm{H}_{\alpha}, 5-\mathrm{H}_{\alpha}\right), 2.52-2.59\left(2 \mathrm{H}, \mathrm{m}, 3^{\prime \prime}-\mathrm{H}\right.$, $\left.6^{\prime \prime}-\mathrm{H}_{\beta}\right), 2.65-2.70\left(2 \mathrm{H}, \mathrm{m}, 3-\mathrm{H}_{\beta}, 5-\mathrm{H}_{\beta}\right), 6.40(1 \mathrm{H}, \mathrm{t}, J=7.6 \mathrm{~Hz},=\mathrm{CH}), 8.47$ $(1 \mathrm{H}, \mathrm{br}, \mathrm{NH}) ;{ }^{13} \mathrm{C}$ NMR $\left(100 \mathrm{MHz}, \mathrm{CDCl}_{3}\right): 17.1\left(3^{\prime \prime}-\mathrm{Me}\right), 21.1\left(5^{\prime \prime}-\mathrm{Me}\right), 25.9$ (C-4), $30.1\left(\mathrm{C}-5^{\prime \prime}\right), 32.4\left(\mathrm{C}-1^{\prime}\right), 34.8\left(\mathrm{C}-6^{\prime \prime}\right), 37.4(\mathrm{C}-3, \mathrm{C}-5), 38.2\left(\mathrm{C}-4^{\prime \prime}\right), 40.4$ (C-3'), $132.5\left(\mathrm{C}-2^{\prime}\right), 138.4\left(\mathrm{C}-1^{\prime \prime}\right), 171.4(\mathrm{C}-2, \mathrm{C}-6), 204.0\left(\mathrm{C}-2^{\prime \prime}\right)$; MS $\left(\mathrm{ESI}^{+}\right)$ $m / z: 286.4[\mathrm{M}+\mathrm{Na}]^{+}$, HRMS $\left(\mathrm{ESI}^{+}\right) \mathrm{m} / z: 286.14111[\mathrm{M}+\mathrm{Na}]^{+}$, calcd mass for $\mathrm{C}_{15} \mathrm{H}_{21} \mathrm{NO}_{3} \mathrm{Na}: 286.14191$.

\section{$N$-methyl-4- $\left[2^{\prime}-\left(3^{\prime \prime}(R), 5^{\prime \prime}(S)-3^{\prime \prime}, 5^{\prime \prime}\right.\right.$-dimethyl-2 $2^{\prime \prime}$ oxocyclohexylidene)ethyl]piperidine-2,6-dione (2)}

Compound 1a $(50 \mathrm{mg}, 0.19 \mathrm{mmol})$, iodomethane $(97 \mathrm{mg}, 0.57 \mathrm{mmol})$ and anhydrous potassium carbonate $(32 \mathrm{mg}, 0.23 \mathrm{mmol}$ ) were added to $3 \mathrm{ml}$ of acetonitrile and stirred at $65^{\circ} \mathrm{C}$ for $10 \mathrm{~h}$. The solution was filtered to remove potassium carbonate and the filtrate was concentrated. The residue was dissolved in $10 \mathrm{ml}$ of ethyl acetate and washed with water. The organic layer was dried over anhydrous sodium sulfate. The solution was filtered to remove any solids and the filtrate was concentrated under vacuum. The residue was purified by chromatography using a silica gel column (100:7 dichloromethane/ methanol) to obtain the title compound $2(48.5 \mathrm{mg}$, yield $92 \%)$ as a white solid.

Physical data for compound 2: m.p. $47-49^{\circ} \mathrm{C} ;[\alpha]_{\mathrm{D}}^{20}=-24.9^{\circ} \mathrm{C}(c=0.25$, $\left.\mathrm{CHCl}_{3}\right)$; IR: $1720(\mathrm{C}=\mathrm{O}), 1671(\mathrm{C}=\mathrm{O}) \mathrm{cm}^{-1} ;{ }^{1} \mathrm{H}$ NMR $\left(400 \mathrm{MHz}, \mathrm{CDCl}_{3}\right) \delta$ : $1.05\left(3 \mathrm{H}, \mathrm{d}, J=5.6 \mathrm{~Hz}, 5^{\prime \prime}-\mathrm{Me}\right), 1.13\left(3 \mathrm{H}, \mathrm{d}, J=6.8 \mathrm{~Hz}, 3^{\prime \prime}-\mathrm{Me}\right), 1.23-1.32(1 \mathrm{H}$, $\left.\mathrm{m}, 4^{\prime \prime}-\mathrm{H}_{\alpha}\right), 1.87-1.92\left(2 \mathrm{H}, \mathrm{m}, 5^{\prime \prime}-\mathrm{H}, 6^{\prime \prime}-\mathrm{H}_{\alpha}\right), 1.96-2.01\left(1 \mathrm{H}, \mathrm{m}, 4^{\prime \prime}-\mathrm{H}_{\beta}\right)$, 2.14-2.26 (3H, m, 1'-H, 4-H), 2.31-2.39 (3H, m, 3"- $\left.\mathrm{H}, 3-\mathrm{H}_{\alpha}, 5-\mathrm{H}_{\alpha}\right), 2.70$ $\left(1 \mathrm{H}, \mathrm{d}, J=13.2 \mathrm{~Hz}, 6^{\prime \prime}-\mathrm{H}_{\beta}\right), 2.80\left(2 \mathrm{H}, \mathrm{d}, J=17.2 \mathrm{~Hz}, 3-\mathrm{H}_{\beta}, 5-\mathrm{H}_{\beta}\right), 3.14(3 \mathrm{H}, \mathrm{s}$, $\mathrm{N}-\mathrm{Me}), 6.35(1 \mathrm{H}, \mathrm{t}, J=7.6 \mathrm{~Hz},=\mathrm{CH}) ;{ }^{13} \mathrm{C} \mathrm{NMR}\left(100 \mathrm{MHz}, \mathrm{CDCl}_{3}\right): 15.7$

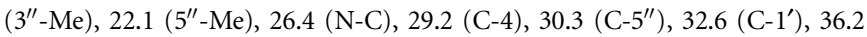
(C-6"), 38.4 (C-3), 38.5 (C-5), 40.8 (C-4"), 44.0 (C-3"), 131.8 (C-2'), 139.1 (C$\left.1^{\prime \prime}\right), 171.8(\mathrm{C}-2, \mathrm{C}-6), 203.5\left(\mathrm{C}-2^{\prime \prime}\right)$; MS $\left(\mathrm{ESI}^{+}\right) \mathrm{m} / z: 278.5[\mathrm{M}+\mathrm{H}]^{+}$and 300.5 $[\mathrm{M}+\mathrm{Na}]^{+}$, HRMS $\left(\mathrm{ESI}^{+}\right) \mathrm{m} / z: 300.15629[\mathrm{M}+\mathrm{Na}]^{+}$, calcd mass for $\mathrm{C}_{16} \mathrm{H}_{23} \mathrm{NO}_{3-}$ Na: 300.15756.

\section{$N$-propyl-4-[2'-(3" $(R), 5^{\prime \prime}(S)-3^{\prime \prime}, 5^{\prime \prime}$-dimethyl-2" -oxo-}

\section{cyclohexylidene)-ethyl]piperidine-2,6-dione (3)}

The title compound was obtained from 1a using a procedure similar to that used to prepare compound 2 . The yield was $17 \%$ as yellow syrup.

Physical data for compound 3: $[\alpha]_{\mathrm{D}}^{20}=-20.6^{\circ} \mathrm{C}\left(c=0.56, \mathrm{CHCl}_{3}\right)$; IR: 1725 $(\mathrm{C}=\mathrm{O}), 1676(\mathrm{C}=\mathrm{O}) \mathrm{cm}^{-1} ;{ }^{1} \mathrm{H}$ NMR $\left(400 \mathrm{MHz}, \mathrm{CDCl}_{3}\right) \delta: 0.88(3 \mathrm{H}, \mathrm{t}$, $J=7.2 \mathrm{~Hz}, \mathrm{~N}-\gamma-\mathrm{Me}), 1.04\left(3 \mathrm{H}, \mathrm{d}, J=6.0 \mathrm{~Hz}, 5^{\prime \prime}-\mathrm{Me}\right), 1.12(3 \mathrm{H}, \mathrm{d}, J=6.8 \mathrm{~Hz}$, $\left.3^{\prime \prime}-\mathrm{Me}\right), 1.25-1.31\left(1 \mathrm{H}, \mathrm{m}, 4^{\prime \prime}-\mathrm{H}_{\alpha}\right), 1.51\left(1 \mathrm{H}\right.$, sext, $\left.J=7.2 \mathrm{~Hz}, \mathrm{~N}-\beta-\mathrm{CH}_{2}\right)$, $1.86-1.92\left(2 \mathrm{H}, \mathrm{m}, 5^{\prime \prime}-\mathrm{H}, 6^{\prime \prime}-\mathrm{H}_{\alpha}\right), 1.96-1.99\left(1 \mathrm{H}, \mathrm{m}, 4^{\prime \prime}-\mathrm{H}_{\beta}\right), 2.11-2.27$ $\left(3 \mathrm{H}, \mathrm{m}, 1^{\prime}-\mathrm{H}, 4-\mathrm{H}\right), 2.31-2.39\left(3 \mathrm{H}, \mathrm{m}, 3-\mathrm{H}_{\alpha}, 5-\mathrm{H}_{\alpha}, 3^{\prime \prime}-\mathrm{H}\right), 2.70(1 \mathrm{H}, \mathrm{d}$, $\left.J=13.2 \mathrm{~Hz}, 6^{\prime \prime}-\mathrm{H}_{\beta}\right), 2.77\left(2 \mathrm{H}, \mathrm{d}, J=16.8 \mathrm{~Hz}, 3-\mathrm{H}_{\beta}, 5-\mathrm{H}_{\beta}\right), 3.71(2 \mathrm{H}, \mathrm{t}, J=7.2 \mathrm{~Hz}$, $\left.\mathrm{N}-\alpha-\mathrm{CH}_{2}\right), 6.34(1 \mathrm{H}, \mathrm{t}, J=7.6 \mathrm{~Hz},=\mathrm{CH}) ;{ }^{13} \mathrm{C}$ NMR $\left(100 \mathrm{MHz}, \mathrm{CDCl}_{3}\right): 11.3$ $(\mathrm{N}-\gamma-\mathrm{C}), 15.7\left(3^{\prime \prime}-\mathrm{Me}\right), 21.2(\mathrm{~N}-\beta-\mathrm{C}), 22.1\left(5^{\prime \prime}-\mathrm{Me}\right), 29.2(\mathrm{C}-4), 30.3\left(\mathrm{C}-5^{\prime \prime}\right)$, $32.5\left(\mathrm{C}-1^{\prime}\right), 36.2\left(\mathrm{C}-6^{\prime \prime}\right), 38.5(\mathrm{C}-3), 38.6(\mathrm{C}-5), 40.8\left(\mathrm{C}-4^{\prime \prime}\right), 41.2(\mathrm{~N}-\alpha-\mathrm{C}), 44.0$ $\left(\mathrm{C}-3^{\prime \prime}\right), 131.9\left(\mathrm{C}-2^{\prime}\right), 139.1\left(\mathrm{C}-1^{\prime \prime}\right), 171.6(\mathrm{C}-2, \mathrm{C}-6), 203.2\left(\mathrm{C}-2^{\prime \prime}\right)$; MS $\left(\mathrm{ESI}^{+}\right)$ $m / z: 306.5[\mathrm{M}+\mathrm{H}]^{+}, 328.5[\mathrm{M}+\mathrm{Na}]^{+}$, HRMS $\left(\mathrm{ESI}^{+}\right) \mathrm{m} / z: 328.18554[\mathrm{M}+\mathrm{Na}]^{+}$, calcd mass for $\mathrm{C}_{18} \mathrm{H}_{27} \mathrm{NO}_{3} \mathrm{Na}$ : 328.18886 .
4-[2'-( $3^{\prime \prime}(R), 5^{\prime \prime}(S)-3^{\prime \prime}, 5^{\prime \prime}$-dimethyl-2" -hydroxylcyclohexylidene)ethyl]piperidine-2,6-dione (4)

To a solution of $1 \mathbf{a}(50 \mathrm{mg}, 0.19 \mathrm{mmol})$ in $3 \mathrm{ml}$ of 1,2-dimethoxyethane (DME), $\mathrm{Zn}\left(\mathrm{BH}_{4}\right)_{2}$-DME ( $4 \mathrm{ml}$, prepared by the method described by Gensler et al. ${ }^{10}$ ) was added slowly. After stirring at room temperature for $24 \mathrm{~h}, 4 \mathrm{ml}$ of water and $1 \mathrm{ml}$ of glacial acetic was added to the reaction mixture. The solution was filtered to remove the precipitate and the filtrate was concentrated under vacuum. The residue was purified by chromatography using a silica gel column (150:10 dichloromethane/methanol). The title compound was collected as a white solid ( $42 \mathrm{mg}$, yield $84 \%$ ).

Physical data for compound 4: m.p. $39-41^{\circ} \mathrm{C} ;[\alpha]_{\mathrm{D}}^{20}=-15.5^{\circ} \mathrm{C}(c=0.47$, $\left.\mathrm{CHCl}_{3}\right)$; IR: $3476(\mathrm{OH}), 3198,3095(\mathrm{NH}), 1681(\mathrm{C}=\mathrm{O}) \mathrm{cm}^{-1} ;{ }^{1} \mathrm{H}$ NMR $\left(400 \mathrm{MHz}, \mathrm{CDCl}_{3}\right) \delta: 0.89-0.92\left(\mathrm{~m}, 4^{\prime \prime}-\mathrm{H}_{\alpha}\right), 0.94\left(3 \mathrm{H}, \mathrm{d}, J=6.0 \mathrm{~Hz}, 5^{\prime \prime}-\mathrm{Me}\right)$, $1.07\left(3 \mathrm{H}, \mathrm{d}, J=6.4 \mathrm{~Hz}, 3^{\prime \prime}-\mathrm{Me}\right), 1.35-1.44\left(3 \mathrm{H}, \mathrm{m}, 3^{\prime \prime}-\mathrm{H}, 5^{\prime \prime}-\mathrm{H}, 6^{\prime \prime}-\mathrm{H}_{\alpha}\right), 1.56(\mathrm{~s}$, $\mathrm{OH}), 1.72-1.76\left(1 \mathrm{H}, \mathrm{m}, 4^{\prime \prime}-\mathrm{H}_{\beta}\right), 2.18-2.22\left(2 \mathrm{H}, \mathrm{m}, 4-\mathrm{H}, \mathrm{1}^{\prime}-\mathrm{H}\right), 2.28-2.34(2 \mathrm{H}$, $\left.\mathrm{m}, 3-\mathrm{H}_{\alpha}, 5-\mathrm{H}_{\alpha}\right), 2.52\left(\mathrm{~d}, J=12.0 \mathrm{~Hz}, 6^{\prime \prime}-\mathrm{H}_{\beta}\right), 2.67-2.72\left(2 \mathrm{H}, \mathrm{m}, 3-\mathrm{H}_{\beta}, 5-\mathrm{H}_{\beta}\right)$, $3.56\left(1 \mathrm{H}, \mathrm{d}, J=10.0 \mathrm{~Hz}, 2^{\prime \prime}-\mathrm{H}\right), 5.42(1 \mathrm{H}, \mathrm{t}, J=6.8 \mathrm{~Hz},=\mathrm{CH}), 7.81(1 \mathrm{H}, \mathrm{br}$, $\mathrm{NH}) ;{ }^{13} \mathrm{C}$ NMR $\left(100 \mathrm{MHz}, \mathrm{CDCl}_{3}\right): 19.1\left(3^{\prime \prime}-\mathrm{Me}\right), 22.1\left(5^{\prime \prime}-\mathrm{Me}\right), 30.9(\mathrm{C}-4)$, $31.8\left(\mathrm{C}-1^{\prime \prime}\right), 33.4\left(\mathrm{C}-5^{\prime \prime}\right), 36.5\left(\mathrm{C}-6^{\prime \prime}\right), 37.3(\mathrm{C}-3), 37.4(\mathrm{C}-5), 41.4\left(\mathrm{C}-3^{\prime \prime}\right), 42.5$ $\left(\mathrm{C}-4^{\prime \prime}\right), 77.9\left(\mathrm{C}-2^{\prime \prime}\right), 113.1\left(\mathrm{C}-2^{\prime}\right), 144.0\left(\mathrm{C}-1^{\prime \prime}\right), 172.2(\mathrm{C}-2, \mathrm{C}-6)$; MS $\left(\mathrm{ESI}{ }^{+}\right)$ $\mathrm{m} / z: 288.3[\mathrm{M}+\mathrm{Na}]^{+}$and $248.3\left[\mathrm{M}-\mathrm{H}_{2} \mathrm{O}\right]^{+}$, HRMS $\left(\mathrm{ESI}^{+}\right) \mathrm{m} / \mathrm{z}: 288.15935$ $[\mathrm{M}+\mathrm{Na}]^{+}$, calcd mass for $\mathrm{C}_{15} \mathrm{H}_{23} \mathrm{NO}_{3} \mathrm{Na}$ : 288.15756.

\section{Anti-HBV assays}

2.2.15 cells in exponential growth phase were seeded into 96-well microplates. A total of $2 \times 10^{4}$ cells per well were used. Test compounds with different concentrations were added, and the cells were cultured in $\mathrm{CO}_{2}$ incubators at $37^{\circ} \mathrm{C}$. The medium was exchanged every 4 days. The positive control (3TC) and untreated cell negative control groups were established. After 8 days, HBV DNA of the lysed cells was extracted. HBV DNA levels were measured using a quantitative Southern blot hybridization analysis approach.

\section{Cytotoxicity assay}

Cytotoxicity induced by the test compounds in the cultures of 2.2 .15 cells was also determined. 2.2.15 cells were seeded into 96-well microplates: $1 \times 10^{5}$ cells per $\mathrm{ml}, 100 \mu \mathrm{l}$ per well. Cells were treated with test compounds at different concentrations. The control was untreated cells. The culture medium was exchanged every 4 days. After 8 days, cell viability levels were examined. $\mathrm{TC}_{50}$ was defined as the concentration that inhibited 50\% cellular growth in comparison with untreated controls and was calculated by Reed and Muench analyses.

\section{ACKNOWLEDGEMENTS}

This work was supported by the National Natural Science Foundation of China (Grant no. 30472076) and a Grant from the Ministry of Science and Technology of China (863 program 2006AA028414). We thank Professors Yang Lv and Yi-kang Si of the Institute of Materia Medica, Chinese Academy of Medical Sciences and Peking Union Medical College for collecting the crystal and $\mathrm{CD}$ data, respectively.

1 Croons, V., Martinet, W., Herman, A. G. \& De Meyer, G. R. Differential effect of the protein synthesis inhibitors puromycin and cycloheximide on vascular smooth muscle cell viability. J. Pharmacol. Exp. Ther. 325, 824-832 (2008).

2 Kit, S., Kurimura, T., De Torres, R. A. \& Dubbs, D. R. Simian virus 40 deoxyribonucleic acid replication. I. Effect of cycloheximide on the replication of SV40 deoxyribonucleic acid in monkey kidney cells and in heterokaryons of SV40-transformed and susceptible cells. J. Virol. 3, 25-32 (1969).

3 Mark, G. E., Taylor, J. M., Broni, B. \& Krug, R. M. Nuclear accumulation of influenza viral RNA transcripts and the effects of cycloheximide, actinomycin D, and alphaamanitin. J. Virol. 29, 744-752 (1979).

4 Watanabe, Y., Kudo, H. \& Graham, A. F. Selective inhibition of reovirus ribonucleic acid synthesis by cycloheximide. J. Virol. 1, 36-44 (1967).

5 Friedman, R. M. \& Grimley, P. M. Inhibition of arbovirus assembly by cycloheximide. J. Virol. 4, 292-299 (1969).

6 Crance, J. M., Biziagos, E., Passagot, J., van Cuyck-Gandré, H. \& Deloince, R Inhibition of hepatitis A virus replication in vitro by antiviral compounds. J. Med. Virol. 31, 155-160 (1990). 
7 Irvine, J. H., Horsfield, J. A., McKinney, C. Z. \& Tate, W. P. A novel strategy to interfere with human immunodeficiency virus type 1 propagation. N. Z. Med. J. 111, 222-224 (1998).

8 Choudhary, S. K. et al. CXCR4 tropic human immunodeficiency virus type 1 induces an apoptotic cascade in immature infected thymocytes that resembles thymocyte negative selection. Virology 352, 268-284 (2006).
9 Schaeffer, H. J. \& Jain, V. K. Investigation of the stereochemistry of cycloheximide. J. Pharm. Sci. 53, 144-147 (1964).

10 Gensler, W. J., Johnson, F. \& David, A. Compounds related to podophyllotoxin. XII. podophyllone and dehydropodophyllotoxin. J. Am. Chem. Soc. 82, 6074-6081 (1960). 